INTERNATIONAL DESIGN CONFERENCE - DESIGN 2018

https://doi.org/10.21278/idc.2018.0342

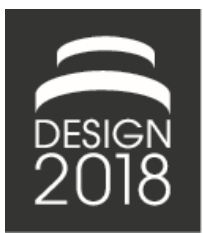

\title{
USE OF ANALOGY IN DESIGN TEAMS: STEPS TOWARDS A COMPUTATIONAL MODEL AND CONCEPTUAL INSIGHTS
}

\author{
V. Singh and H. Casakin
}

\begin{abstract}
This paper presents a computational framework to study the effects of the use of analogy in design teams on team cohesion and team collaboration. The paper also reports on the new conceptual insights and lessons learnt while developing the model. The lessons learnt are particularly useful, because the use of computational models to study social aspects of team work in design research is still a fairly recent development, and the challenges and benefits in the process of formalizing abstract concepts into computational details are rarely discussed in the literature.
\end{abstract}

Keywords: agent based modelling, design teams, simulation based design, collaborative design, analogy in design

\section{Introduction}

While the use of analogy in design and design creativity has been researched for some time, it is only recently that researchers have begun to investigate the effects of analogizing in design on team dynamics (Ball and Christensen, 2009; Casakin et al., 2015). Casakin et al. (2015) studied factors such as analogical distance and analogical purpose, which were found to have an effect on team collaboration and team cohesion. These findings were based on qualitative analysis of one set of empirical data collected during a design session. Therefore, it is desirable that additional data and further research is conducted to validate those findings, and build on them. However, as Singh and Casakin (2015) pointed out, repeating such empirical studies is demanding in terms of resources and time, and several challenges exist in studying behavioural aspects of teams like team collaboration and team cohesion. For instance, main questions are how to control these variables in the experiments, and how to assure the reliability of the findings; or with a limited number of empirical studies, how to generalize the findings. Similarly, during these empirical studies additional parameters and open questions that require further investigation and validation are necessary.

Important work on computational analogy was done by Fu et al. (2013), who introduced a methodology for discovering structure in design repository databases. Using a Bayesian model combined with Latent Semantic Analysis for identifying structural form in data, the main goal of the study was to develop a computational design stimulation tool that enables designers to discover and gain automatic access to cross domain analogical stimuli from a design repository.

Significant work on cognitive-based computational modelling was carried out by McComb et al. (2015) dealing with design teamwork. The investigation presented a computational framework that simulates team-based engineering design using agents that solve engineering problems. The framework was used to test and evaluate design strategies, as well as to represent aspects of designer behaviour. The model proved its utility by simulating cognitive studies about the behaviour of human design teams. Further 
work on cognitive-based computational team modelling explored the formation of mental models during team problem-solving regarding interaction structure (Dionne et al., 2010) and agent memory (Sayama, et al., 2011). Another agent-based design team model studied the influence of team composition and task complexity on the creation of transactive memory (Singh, et al., 2012).

Based on the above studies, Singh and Casakin (2015) considered the use of agent based computational simulations as complementary research methods that could facilitate the research enquiry. Consistent with previous research on computational social science (e.g. Lant, 1994; Carley, 1994; Levitt et al., 2005) preliminary studies can be carried out to test and generate hypotheses of interest, which can then be used to conduct further empirical studies. While agent based computational models require simplification of the experimental scenarios, they are particularly useful in terms of their repeatability, control, scalability and the ability to support longitudinal studies. However, there should be an independent method to assess the reliability and effectiveness of a computational model used for studying social behaviour (Axtell et al., 1996; Axelrod, 1997; Levitt et al., 2005). Validation methods such as equivalency test (Axtell et al., 1996), and recognizable social behaviour from the simulation models (Carley and Newell, 1994) have been proposed to assess the utility of the developed computational models.

Singh and Casakin (2015) proposed a conceptual framework for developing a computational model that could support studying the use of analogy in design teams and how it affects team collaboration and team cohesion. The proposed computational framework builds on theories, and findings based on empirical evidence reported in recent literature (Casakin et al., 2015; Ball and Christensen, 2009; Christensen and Schunn, 2007). This paper presents a further development of the framework proposed by Singh and Casakin. It reports the steps towards which the computational model has evolved, and discusses conceptual insights gained, as well as lessons learnt, in developing the framework.

\section{Background}

\subsection{Analogical reasoning in design and design teams}

Analogical reasoning is broadly explained as the access, retrieval, and transfer of prior knowledge from a familiar situation (the source) to a situation that needs to be elucidated (the target) (e.g. Ball et al., 2004; Ball and Christensen, 2009; Casakin, 2004, 2010; Casakin and Goldschmidt, 1999). Analogical reasoning enables to see a new situation in terms of a known one (Holyoak and Thagard, 1995).

Even though design is frequently carried out in team, less work has been carried out to explore how the use of analogy and analogy-related cognitive strategies support teamwork (Rousseau et al., 2006). More recently researchers such as Christensen and Schunn (2007), and Stacey et al. (2007) begun to study the use of analogy in design teams. A common finding was that analogizing is regularly used by design teams in a variety of design activities (Ball and Christensen, 2009). Some of the key findings so far about the role of analogy in design and design teams include:

- Analogies can be categorized into 'within-domain' (source and target domains are close) and 'between-domain' (source and target domains are distant). The use of analogy was found to be related to expertise; whereas expert designers tend to retrieve analogies from between-domain sources, novices have a tendency to use within-domain sources (Casakin, 2004).

- Analogical reasoning can play a role in attaining mutual understanding among design team members. The use of analogy can broaden and enrich the idea generation process, and facilitate communicative exchanges between team members (Stempfle and Badke-Schaub, 2002).

- Christensen and Schunn (2007) showed that analogising was largely used by teams for three major functions, including problem identification, solution generation, and explaining. It was found that: (1) within-domain analogies were frequently employed for problem identification; (2) betweendomain analogies prevailed during explanation activities; and (3) both within-domain and between-domain analogies were equally employed for solution generation.

- Ball and Christensen (2009) showed that analogy was also used for function finding, that is, retrieving new functions for the target design.

Thus, four primary types of analogical purposes have been identified: problem identification, solution generation, explanation, and function finding. Casakin et al. (2014) found that all four types of 
analogical purpose had a likely association with team cohesion, while solution generation and function finding had a stronger association with team collaboration.

\subsection{Team cohesion and team collaboration}

Communication exchanges between team members was seen to affect team cohesion and team collaboration. Social, cultural, and emotional aspects of teamwork were investigated in relation to team cohesion (Badke-Schaub et al., 2007). Members of a cohesive design team positive feelings bonding to one another, and to the group as a whole (Goodman et al., 1987). Team cohesion prevents poor climate, tensions, frictions and group fragmentation (Boos et al., 2011). A cohesive group has tendency to be in unity at the time that they work towards a common shared goal (Carron and Brawley, 2000; Beal et al., 2003). The creation and maintenance of a cohesive climate (Badke-Schaub et al., 2011) in the design team imply that their members will desire to stay together in order to achieve a work. In a recent study, Casakin and Badke-Schaub (2017) operationalized team cohesion in terms of appreciation, confirmation, rejection, and help provided to other members. These researchers found that team cohesion resulted in an essential parallel activity to the work on the design task, ensuring positive feedback in the group that enable to handle critical issues without deriving into conflicts. Whereas team cohesion is concerned social, and mainly emotional aspects of the group, team collaboration is referred to information exchange for the sake of achieving successful design outcomes. Kleinsmann and Valkenburg (2007) defined team collaboration as actors integrating and communicating their knowledge about the design content and process in order to attain a 'shared understanding', for the creation of a novel design outcome. In this sense, the quality of the design outcome has shifted from the activity of the individual designer to the interaction of several designers working as a team, as well as to the collaboration of different multi-disciplinary teams (Loch et al., 1996). Team collaboration builds on the need of exchanges of knowledge, skills, and experiences (Casakin and Badke-Schaub, 2013). Collaboration encompasses a mutual understanding of the task, as well as organizing and synchronizing those processes that define roles and responsibilities of the team members. Assigning roles and taskrelated activities that are suitable to the team members' competencies early in the process, enhances the chances of accomplishing goals effectively.

In what ways the use of analogy can contribute to enhance team cohesion and collaboration as a social group has yet to be explored. Casakin et al. (2015) found that analogical purpose had strong association with team cohesion, whereas solution generation and function finding was associated with team collaboration.

\section{Research framework}

In their research framework, Singh and Casakin (2015) identify the following parameters and variables (see Table 1).

Table 1. List of independent, mediating and dependent variables and parameters in the framework

\begin{tabular}{|l|l|ll|}
\hline Independent variables & Analogical type/distance & $\begin{array}{l}\text { i) } \\
\text { ii) }\end{array}$ & $\begin{array}{l}\text { within-domain; } \\
\text { between-domain }\end{array}$ \\
\hline & Team composition/ & i) & level of multi-disciplinarity; \\
& Expertise of team members: & ii) & level of expertise (expert/novice); \\
\hline & Team size & i) & small; \\
& & $\begin{array}{l}\text { ii) } \\
\text { medium; }\end{array}$ & large \\
\hline Mediating variable & Analogical purpose & i) & problem identification; \\
& & ii) & function; \\
& & iii) & $\begin{array}{l}\text { solution generation finding; } \\
\text { explanation }\end{array}$ \\
\hline Dependent variables & Teamwork & i) & team collaboration; \\
& & ii) & team cohesion \\
\hline
\end{tabular}


Once the computational model is developed and validated, the model can be used to generate and test preliminary hypotheses related to analogy and the interaction of the design team. For example, it can be studied how the use of analogy changes and influences team cohesion and team collaboration with varying team composition; that is, varying ratio of experts and novices in a team. Similarly, another issue is whether an increase in the number of experts will make a difference in the team cohesion. The effect of these and other parameters on design teamwork such as team size can be explored with computational models.

\section{Computational framework}

This section elaborates on the proposed computational framework, including aspects such as task and knowledge representation, analogy, team interaction and key parameters and measures. The design team is viewed as a collection of social agents, who interact through message passing to collaborate and solve a given task. Some details of the message passing protocols, the choice of interaction vocabulary and how that could facilitate the assessment of team collaboration and team cohesion in the simulated environment are presented.

\subsection{Task and knowledge representation}

A multi-disciplinary knowledge base is predefined in the agent environment, such that it allows simulating scenarios that use within-domain and between-domain analogies. Multi-disciplinarity is represented as a finite set of knowledge domains available to the design society, discretely classified as ' $\mathrm{m}$ ' disciplines. We assume that the size of the design/knowledge space in a given discipline can be represented as a discrete set of ' $n$ ' concepts. Each design solution is a set of compatible concepts belonging to one or more disciplines. The design space can therefore be generated out of permutations and combinations of elements from an $\mathrm{m} \times \mathrm{n}$ matrix. For most problems, alterative design solutions can be achieved through analogy (simulated), whereby one or more concepts from a known solution can be replaced with equivalent concepts from the same or other disciplines.

The concepts in the different domains can be categorized either as a function, behaviour or a structure (FBS) (for further explanations about the FBS framework see Gero and Kannengiesser, 2002; 2004). Thus, establishing an analogy can be based on functional, structural or behavioural similarities. For a solution to be valid a compatible set of function, behaviour and structure concepts must be present. The analogical distance between any two concepts is calculated only when they belong to the same category. That is, analogical distance can be calculated between two functional concepts, two behavioural concepts, or two structural concepts. However, analogical distance cannot be calculated by combining any of these categories; i.e., between a functional and a structural concept, a functional and a behavioural concept, and so on.

Based on their own experience, designers may come with different solutions for the same problem. The combination of concepts by a designer may vary according to his or her background and expertise. The present model assumes that expert designers will establish between-domain analogies by choosing a set of concepts from different disciplines, while novices will tend to be constrained by within-domain analogies, and consequently they will probably select fewer and less original design concepts to deal with a problem.

Analogical distance is represented by considering (1) distance between two domains or two disciplines, and (2) distance between two concepts belonging to the same domain or discipline. In our model, we assume that the proximity between any two disciplines, $\mathrm{m}^{\mathrm{i}}$ and $\mathrm{m}^{\mathrm{j}}$, for the purpose of representing analogical distance can be calculated as $|\mathrm{j}-\mathrm{i}|$. Similarly, the analogical distance between any two concepts ${ }^{\mathrm{n}} \mathrm{k}_{\mathrm{a}}$ and ${ }^{\mathrm{n}} \mathrm{k}_{\mathrm{b}}$, in the $\mathrm{k}^{\text {th }}$ discipline can be calculated as $|\mathrm{b}-\mathrm{a}|$. By combining these two ways of representing the distance between disciplines and the distance between concepts, the analogical distance between any two concepts ${ }^{\mathrm{n}} \mathrm{k}_{\mathrm{a}}$ and ${ }^{\mathrm{n}} \mathrm{l}_{\mathrm{b}}$ across two disciplines $\mathrm{k}$ and 1 , can be calculated as $|\sqrt{ }[(1-\mathrm{k}) 2+(\mathrm{b}-\mathrm{a}) 2]|$.

Analogical purpose: The four types of analogical purpose, namely solution generation, function finding, explanation, and problem identification, will be represented as pre-coded objectives. Agents' action at any given instance is a reaction to the objectives at that instance. The objectives at any given instance will be a probabilistic function, also contingent on the actions and objectives in the preceding instances. 
Thus, the solutions and task related interactions will remain non-deterministic, based on Monte Carlo results.

\subsection{Agents and parameters}

- Level of multi-disciplinarity of an agent is modelled as the number of disciplines that an agent has knowledge of.

- Expertise in a given discipline is modelled as the number of concepts in a discipline that are known to an agent.

- The combination of the level of multi-disciplinarity and level of expertise allows for multiple expertise profiles to be represented.

For example, expert designers that are specialists can be assumed to know a wide range of concepts in a given domain, but may know very few concepts from other domains. Similarly, experts that are generalists can be assumed to know concepts from a wide range of domains, but may know only some of the concepts from each of these domains. On the other hand, novices may know just a few concepts. Besides knowledge of a number of concepts, it is possible to extend the agent model features with additional parameters such as apriori knowledge of functional, behavioural and structural (FBS) concepts, and the analogical distance between these concepts. Thus, it is possible to distinguish between the breadth of FBS concepts known in one or more specific domains by an agent, and the extent of known FBS analogical mappings that the agent can establish within and between domains. Nonetheless, following the bottom-up process of developing the computational model, the complexity of the model and number of parameters will be increased incrementally.

\subsection{Team representation and parameters}

In the present model, design teams will be represented according to:

- Team composition: the combination of levels of multi-disciplinarity and levels of expertise for individual agent profiles also allows creating various team compositions, and this enables testing various research hypotheses. For example, what kind of team composition might be more conducive to team cohesion?

- Team size: this is a default parameter, though it may not necessarily be independent of team composition. For example, while small teams can be composed entirely of experts or novices, it is rarely the case that a large team is composed only by experts. Often, large teams have a combination of experts and novice members. Similarly, large teams are often composed of specialists as well as generalists. Therefore, assumptions about team size and team composition will be based on typical scenarios observed in practice.

The ability to run a wide range of simulation scenarios for analogy-based design collaboration with parameters such as team composition and team size is particularly the kind of advantage computational approaches allow, which is difficult to manage in empirical studies with human subjects. Consequently, most studies that have been reported on analogy-based design collaboration are conducted with a small team size, and with limited possibility to change the team composition. In addition, such empirical studies allow for limited control on the prior knowhow and expertise of the team members, which can get difficult to track as the team size increases. In contrast, computational simulations can allow team sizes to vary from small groups to extremely large teams, with varying team structures and additional attribute, while still allowing for control on agent attributes and their prior knowledge, and track the changes in the knowledge of each of the individual agents.

\subsection{Modelling collaboration and teamwork}

For the agents to collectively solve a problem, other aspects of team interaction need to be modelled such as the mechanisms for knowledge sharing and information exchange. These mechanisms are by themselves not necessarily to be used as variables in this research, but these assumptions are necessary to model and simulate conditions under which the desired studies can be conducted. By keeping these modelling assumptions constant, their effects are nullified across the simulation cases. In future studies, 
however, it should be possible to experiment with these mechanisms as well, using them as simulation parameters.

\subsubsection{Transactive memory}

In order for agents in a team to be able to interact with other agents in the same team, and collectively solve the given task, they need to know 'who knows what'. That is, agents need to have a transactive memory of the team (Wegner, 1995).

The transactive memory of an agent is included within the agent mental model (AMM), which is represented as $m \times p$ matrix, where ' $m$ ' is the number of disciplines, and ' $p$ ' is the number of agents in the team. This approach builds on Singh et al.'s (2013a) implementation of AMM, where each element of the matrix is a vector representing the corresponding agent's multi-disciplinarity profile as learnt by the agent. Understanding the profile of the team members allows an agent to adjust the communication and choice of analogies based on the multi-disciplinarity and expertise of the other agents. Agents in the team can begin with a partly pre-learnt AMM for other agents, and as the interaction evolves, they can update or modify the AMM. The pre-learnt AMM can be assumed, simulating either that the team members have a prior-familiarity (Singh et al., 2009) or that they make generic assumptions based on disciplinary backgrounds of the team members.

\subsubsection{Interaction between agents}

Agents in the team interact through direct message passing. Messages can be one-to-one, or one-tomany. One-to-many messages can be used to simulate a group discussion, which is typical of small teams that will be simulated in the initial cases.

A comprehensive list of the diverse types of messages and message vocabulary that will suffice to simulate the desired cases will be pre-coded in the model. To develop such a list of messages it is important to identify the communicative actions required for achieving an analogy-based design activity. Some of the steps required for this include:

- A problem is identified and communicated, which outlines the requirements of a target solution. The requirements can be stated in terms of functional, behavioural or structural concepts (FBS). Thus, the message protocol needs to clearly identify the requirement type.

- An analogy-based solution is proposed identifying the source. Analogy can be used for solution identification at various levels of problem decomposition, which can be viewed in terms of functions and sub-functions. The functional decomposition will also be applicable to both behavioural as well as structural concepts.

- The receiver of the proposed analogy-based solution may or may not understand the analogy, in which case an explanation is sought.

- Explanations are expected to foster the formation of a shared understanding and creation of common ground. In the process, learning takes place, and new knowledge and concepts are acquired. Learning can occur not only for the interacting agents, but also for the other agents in the team who might be observing the interaction between any two agents. Different modes of social learning can be implemented based on Singh et al. (2009, 2013b).

- Giving and seeking explanation may iterate multiple times until either the understanding takes place, or the proposal is replaced by another one. It is likely that additional analogies are used to facilitate the identification of shared concepts and common ground. This can lead to mapping of analogies that were otherwise not comprehensible to one of more of the interacting agents.

- After each episode of analogy negotiation and communication, the interacting agents or the team either select or reject a solution, and the interaction moves into the next phase, which could be to another function or sub-function. As stated earlier, these interactions may also proceed in terms of behavioural or structural analogy.

While the above list shows an indicative set of steps required for analogy-based design communication, the communicative actions can be broadly classified corresponding to design task, design process and team cohesion. Table 2 shows classification codes based on Casakin et al. (2015). 
Table 2. Communicative actions in analogy-based design

\begin{tabular}{|c|c|}
\hline Type & Action: Description \\
\hline \multirow[t]{6}{*}{ Task } & $\begin{array}{l}\text { PD-Problem definition: Definitions that are considered in order to formulate and } \\
\text { define the problem }\end{array}$ \\
\hline & $\begin{array}{l}\text { SI-New solution idea: Stating a new idea or a new solution, or developing new aspects } \\
\text { of an earlier solution idea }\end{array}$ \\
\hline & SA-Solution analysis: Analysis of a solution idea or part of it \\
\hline & $\begin{array}{l}\text { SAE-Solution evaluation: Assessment of a solution idea by focusing on its value and } \\
\text { feasibility }\end{array}$ \\
\hline & $\begin{array}{l}\text { SAX-Explanation: Clarification of aspects and questions related to design issues, i.e., } \\
\text { user, technical, or budget. }\end{array}$ \\
\hline & SD-Solution decision: A final and definitive decision for a solution \\
\hline \multirow[t]{3}{*}{ Process } & PL-Planning: Aspects related to when to proceed and what to do \\
\hline & $\begin{array}{l}\text { PR-Procedures: How to proceed to deal with the task, what strategies and methods } \\
\text { should be used }\end{array}$ \\
\hline & $\begin{array}{l}\text { RF-Reflection: What the team has been doing so far, and what aspects have been } \\
\text { influential in the design }\end{array}$ \\
\hline \multirow[t]{4}{*}{ Team cohesion } & $\begin{array}{l}\text { AP-Appreciation: Approval of other team members in support of a problem definition, } \\
\text { a solution idea, or an explanation }\end{array}$ \\
\hline & C-Confirmation: Positive statements endorsing other team members' statements \\
\hline & RJ-Rejection: Disapproval of other team members about an idea, or an explanation \\
\hline & H- Help: Assistance provided to other team members \\
\hline
\end{tabular}

Figure 1 shows an example of a potential interaction sequence, described using the task related codes listed in Table 2 (Based on Casakin and Badke-Schaub, 2017).

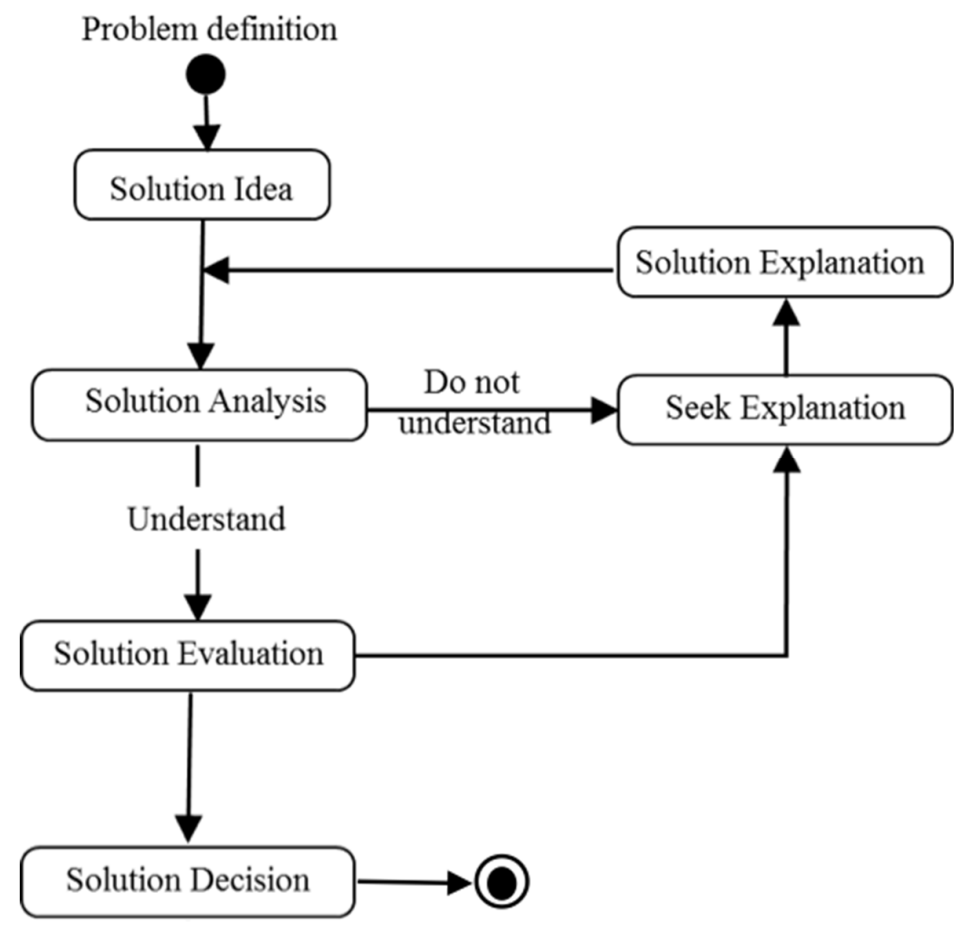

Figure 1. Example communication sequence described in terms of task-related codes 
The sequence of messages and contents at any given instance will be a probabilistic function of how the problem and solutions emerge. Consequently, the message patterns will remain nondeterministic.

- Protocol for message passing: The message passing across the agents will be based on FIPAACL (Foundation for Intelligent Physical Agents-Agent Communication Language) protocols, built on top of Singh et al.'s (2010) implementation of a design team interaction simulator, developed using JADE (Java Agent Development Environment) platform.

\subsection{Measuring outcomes: Team collaboration and team cohesion}

Casakin et al. (2015) measured team collaboration by categorizing each episode of design activity and assessing whether team collaboration occurred in that instance, depending on whether more than one agent contributed to the objectives. Similarly, they assumed team cohesion to occur in any given episode of design activity when a shared understanding of an analogy, or an analogical distance across the discussed concepts between two or more agents is observed.

Thus, for measuring both team collaboration and team cohesion, the messages exchanged between the agents in each design episode are measured using a coding scheme similar to the one listed in table 1. Since the computational model will implement a message passing vocabulary based on the same coding scheme, this research will use the same protocol analysis technique to measure team collaboration and team cohesion.

\section{Discussion}

Building on Singh and Casakin (2015), this paper provides preliminary details on how the computational framework will be developed towards an agent-based simulation platform.

As part of developing the computational framework and detailing towards an implementable simulation tool, several research design decisions are made that provide new insights and raise new questions about analogizing in design teams. In transitioning from subjective understanding of theoretically abstract concepts to objective parameters, several assumptions need to be made. Similarly, in implementing and choosing the mechanisms or causal relationships that can allow computational actions and functions to be realized, theoretical gaps in the current understanding of analogizing are recognized, providing new research questions and research hypotheses of interest. Therefore, the rest of this section centres on: (1) clarifying the main focus of the proposed computation model; that is, what can and cannot be studied using the proposed model; and (2) those aspects of analogizing that were assumed for implementation, but for which there was limited prior literature, indicating a gap to be addressed in empirical studies as well.

One major issue is focuses on social effects of analogy in design and team dynamics, rather than on design solutions: Most studies on analogy centre on design cognition aspect of analogy such as creativity and design outcomes, whereas social aspects like team dynamics tend to be overlooked. In particular, computational discussion on analogy so far has primarily been directed towards simulating analogical processes (e.g., Gero et al., 2008; Gero and Kannengiesser, 2012), or understanding analogical processes from cognition and intelligence viewpoints (e.g. Chan et al, 2011; Fu et al, 2013). Rather than studying the effects of analogy on design outcomes, the present research aims at focusing on the social effects of analogy. Hence, it is acceptable that the proposed model has a pre-coded design and knowledge space, so that all the design solutions generated during the team activity are limited to this design space.

Another central theme is concerned with nested analogies for convergence towards a common understanding: In current design literature, four types of analogical purposes were observed including: problem identification, solution generation, explanation and function finding. No matter what the purpose of analogy in the design team is, an analogy used by one team member must be comprehensible to the other. This raises an interesting situation, which was identified while assessing potential interaction scenarios that are likely to occur during the simulated design sessions. A model characterized by a team with different disciplinary backgrounds, may include both, agents with common shared concepts, and agents possessing mutually exclusive concepts. Employing a mutually exclusive concept to analogise, may lead an agent to look for additional analogies as an attempt to find a shared concept 
that would be comprehensible to the others. In this exchange process, it is likely that multiple analogies and concepts will emerge. The production of nested analogies, which can be considered as part of the explanation category, is a scenario that has not been adequately explored in the current design literature yet.

An additional but not less important topic deals with the correlation between the level of multidisciplinarity and the level of expertise: In the design literature, it is often stated that multidisciplinary teams are desirable, but the description of multi-disciplinarity is typically limited to the formal or professional background of the team members. The team profile is rarely defined to a level of detail that could help team managers go beyond a general understanding of the multidisciplinary team composition, and in most cases the level and type of expertise of the members remain unknown. For example, two architects that have the same years of experience may have very different multidisciplinary and expertise profiles.

Similarly, the discussion on the difference in analogizing between experts and novices rarely deals with their level of multi-disciplinarity. However, we maintain that analogical reasoning cannot be discussed sufficiently without going into the details of multi-disciplinarity and expertise of the design team.

Another insight gained during development of the interaction scenarios was the recognition of the potential significance of the functional, behavioural and structural concepts: it is possible that agents seek to establish an analogy based on functional, behavioural or structural concepts. Consequently, related hypotheses have been identified that can be useful for further empirical investigations, once preliminary patterns of analogical reasoning have been established from simulation results. For example, a question could be whether analogies based on behavioural concepts provide a wider range of alternative solutions compared to those analogies based on functional or structural concepts.

A final key insight that emerged while developing the present computational framework consisted in identifying gaps in current design literature on the above-mentioned topics: There is a need for a more objective and deeper understanding of multi-disciplinarity in teams, and its relationship with the level of expertise, both in design in general, and in the use of analogy specifically.

\section{Conclusion}

This paper discussed the development of a computational framework intended to provide the basis for an agent-based simulation model to study the role of analogizing in design team interactions. Unlike other computational approaches to study analogy in design, this framework specifically focuses on social aspects of analogising in design teams, especially its effect on team cohesion and team collaboration. The iterative development of the computational framework led to new theoretical and conceptual insights about the problem context. Most notable insights were the lack of objective description of multi-disciplinarity in design literature, and the need to explore its relation with expertise in the context of analogy in design teams.

\section{References}

Axelrod, R. (1997), "Advancing the art of simulation in the social sciences", In: Conte, R., Hegselmann, R. and Terna, P. (Eds.), Simulating Social Phenomena, Springer Berlin, Germany, pp. 21-40. https://doi.org/10.1002/(SICI)1099-0526(199711/12)3:2\%3C16::AID-CPLX4\%3E3.0.CO;2-K

Axtell, R., Axelrod, R., Epstein, J. and Cohen, M. (1996), “Aligning simulation models, a case study and results", Computation and Mathematical Organization Theory, Vol. 1 No. 2, pp. 123-141. https://doi.org/10.1007/BF01299065

Badke-Schaub, P., Neumann, A. and Lauche, K. (2011), “An observation-based method for measuring the sharedness of mental models in teams”, In: Boos, M., Kolbe, M., Kappeler, P.M. and Ellwart, T. (Eds.), Coordination in Human and Primate Groups, Springer-Verlag Berlin, Germany, pp. 177-197. https://doi.org/10.1007/978-3-642-15355-6_10

Badke-Schaub, P., Neumann, A., Lauche K. and Mohammed, S. (2007), "Mental models in design teams: A valid approach to performance in design collaboration?", CoDesign, Vol. 3 No. 1, pp. 5-20. https://doi.org/10.1080/15710880601170768 
Ball, L.J. and Christensen, B.T. (2009), “Analogical reasoning and mental simulation in design: Two strategies linked to uncertainty resolution", Design Studies, Vol. 30 No. 2, pp. 567-589. https://doi.org/10.1016/j.destud.2008.12.005

Ball, L.J., Ormerod, T.C. and Morley, N.J. (2004), "Spontaneous analogising in engineering design: a comparative analysis of experts and novices", Design Studies, Vol. 25 No. 5, pp. 495-508. https://doi.org/10.1016/j. destud.2004.05.004.

Beal, D.J., Cohen, R.R., Burke, M.J. and Mclendon, C.L. (2003), "Cohesion and performance in groups: a meta-analytic clarification of construct relations", Journal of Applied Psychology, Vol. 88 No. 6, pp. 9891004. https://doi.org/10.1037/0021-9010.88.6.989

Boos, M., Kolbe, M. and Strack, M. (2011), “An inclusive model of group coordination”, In: Boos, M. Kolbe, M., Kappeler, P.M. and Ellwart, T. (Eds.), Coordination in Human and Primate Groups, Springer, pp. 11-35. https://doi.org/10.1007/978-3-642-15355-6_2

Carley, K. (1994), “Sociology: Computational organization theory”, Social Science Computer Review, Vol. 12 No. 4, pp. 611-624. https://doi.org/10.1177/089443939401200410

Carley, K.M. and Newell, A. (1994), “The nature of the social agent”, Journal of Mathematical Sociology, Vol. 19 No. 4, pp. 221-262. https://doi.org/10.1080/0022250X.1994.9990145

Carron, A.V. and Brawley, L.R. (2000), "Cohesion: conceptual and measurement issues", Small Group Research, Vol. 31 No.1, pp. 89-106. https://doi.org/10.1177/1046496412468072

Casakin, H. (2004), "Visual analogy as a cognitive strategy in the design process: Expert versus novice performance", Journal of Design Research, Vol. 4 No 2, pp. 197-217. http://dx.doi.org/10.1177/104649640003100105

Casakin, H. (2010), "Visual analogy, visual displays, and the nature of design problems: The effect of expertise", Environment and Planning B: Planning and Design, Vol. 37 No. 1, pp. 170-188. https://doi.org/10.1068/b35073

Casakin, H. and Badke-Schaub, P. (2013), "The psychology of creativity: Mental models in design teams", In: Antonietti, A., Colombo, B. and Memmert, D. (Eds.), Psychology of creativity, Nova Science Publishers, New York, pp. 167-180.

Casakin, H. and Badke-Schaub, P. (2017), "Sharedness of team models in the course of design-related interactions between architects and clients", Design Science., Vol. 3 e14. https://doi.org/10.1017/dsj.2017.15

Casakin, H. and Goldschmidt, G. (1999), "Expertise and the visual use of analogy: implications for design education”, Design Studies, Vol. 20 No. 2, pp. 153-175. https://doi.org/10.1016/S0142-694X(98)000325

Casakin, H., Ball, L., Christensen, B.T. and Badke-Schaub, P. (2015), "How do analogizing and mental simulation influence team dynamics in innovative product design?", Artificial Intelligence for Engineering Design, Analysis and Manufacturing (AIEDAM), Vol. 29 No. 2, pp. 173-183. https://doi.org/10.1017/S0890060415000050

Chan, J., Fu, K., Schunn, C., Cagan, J., Wood, K. and Kotovsky, K. (2011), “On the benefits and pitfalls of analogies for innovative design: Ideation performance based on analogical distance, commonness, and modality of examples", Journal of Mechanical Design, Vol. 133 No. 8, pp. 081004. https://doi.org/10.1115/1.4004396

Christensen, B.T. and Schunn, C.D. (2007), "The relationship of analogical distance to analogical function and pre-inventive structure: The case of engineering design", Memory and Cognition, Vol. 35 No. 1, pp. 29-38. https://doi.org/10.3758/BF03195939

Dionne, S.D., Sayama, H., Hao, C. and James, B. (2010). "The role of leadership in shared mental model convergence and team performance improvement: an agent-based computational model", The Leadership Quarterly, Vol. 21 No. 6, pp. 1035-1049. http://dx.doi.org/10.1016/j.leaqua.2010.10.007.

Forsyth, D.R. (2010), Group Dynamics, 5th ed., Cengage Learning Wadsworth.

Fu, K., Chan, J., Cagan, J., Kotovsky, K., Schunn, C. and Wood, K. (2013), “The meaning of 'near' and 'far': The impact of structuring design databases and the effect of distance of analogy on design output", Journal of Mechanical Design, Vol. 135 No. 2, pp. 021007. https://doi.org/10.1115/1.4023158

Gero J.S. and Kannengiesser, U. (2002), “The situated function-behaviour-structure framework", Artificial Intelligence in Design '02, Kluwer Academic Publishers, Dordrecht, pp. 89-104. https://doi.org/10.1007/978-94-017-0795-4_5 
Gero J.S. and Kannengiesser, U. (2004), "The situated function-behaviour-structure framework", Design Studies, Vol. 25 No. 4, pp. 373-391. https://doi.org/10.1016/j.destud.2003.10.010

Gero, J.S. and Kannengiesser, U. (2012), "Representational affordances in design, with examples from analogy making and optimization", Research in Engineering Design, Vol. 23 No. 3, pp. 235-249. https://doi.org/10.1007/s00163-012-0128-y

Gero, J.S., Grace, K. and Saunders, R. (2008), “Computational analogy making in designing: A process architecture", Proceedings of CAADRIA 2008, Pimniyom Press, Chiang Mai, Thailand, pp. 153-160.

Goodman, P.S., Ravlin, E. and Schminke, M. (1987), “Understanding groups in organizations”, In: Staw, B.M. and Cummings, L.L. (Eds.), Research in organizational behavior, JAI, Greenwich, CT, pp. 121175.

Holyoak, K.J. and Thagard, P. (1995), Mental leaps: analogy in creative thought, MIT Press, Cambridge, MA. https://doi.org/10.1207/s15327868ms1201_8

Kleinsmann, M.S. and Valkenburg, R. (2007), "Why do(n't) actors in collaborative design understand each other? An empirical study towards a better understanding of collaborative design", CoDesign, Vol. 3 No. 1, pp. 59-73. https://doi.org/10.1080/15710880601170875

Lant, T.K. (1994), "Computer simulation of organizations as experimental learning systems: Implications for organization theory", In: Carley, K.M. and Prietula, M.J. (Eds.), Computational Organization Theory, Lawrence Erlbaum Associates, New Jersey, pp. 195-215.

Levitt, R.E., Orr, R.J. and Nissen, M.E. (2005), Validation of the virtual design team (VDT) computational modelling environment, Standford University.

Loch, C., Stein, L. and Terwiesch, C. (1996), "Measuring development performance in the electronics industry", Journal of Product Innovation Management, Vol. 13 No. 1, pp. 3-20. https://doi.org/10.1016/0737-6782(95)00089-5

McComb, C., Cagan, J. and Kotovsky, K. (2015), "Lifting the veil: Drawing insights about design teams from a cognitively-inspired computational model", Design Studies, Vol. 40 No. 3, pp. 119-142. https://doi.org/10.1016/j.destud.2015.06.005

Rousseau, V., Aube, C. and Savoie, A. (2006), "Teamwork behaviors: A review and an integration of frameworks", Small Group Research, Vol. 37 No. 5, pp. 540-570. https://doi.org/10.1177/1046496406293125

Sayama, H., Farrell, D.L. and Dionne, S.D. (2011), “The effects of mental model formation on group decision making: an agent-based simulation”, Complexity, Vol. 16 No. 3, pp. 49-57. https://doi.org/10.1002/cplx.20329

Singh, V. and Casakin, H. (2015), "Developing a computational framework to study the effects of use of analogy in design on team cohesion and team collaboration", Proceedings of the 20th International Conference on Engineering Design (ICED15), Human Behavior in Design, Design Education, Milan, Italy, July 27 - 30, 2015, pp. 101-110.

Singh, V., Dong, A. and Gero, J.S. (2009), "Effects of social learning and team familiarity on team performance", Proceedings of the Spring Simulation Multicongference, San Diego, California, March 22 - 27, 2009. https://doi.org/10.1145/1639809.1639816

Singh, V., Dong, A. and Gero, J.S. (2012), “Computational studies to understand the role of social learning in team familiarity and its effects on team performance", CoDesign, Vol. 8 No. 1, pp. 25-41. http://dx.doi.org/10.1080/15710882.2011.633088.

Singh, V., Dong, A. and Gero, J.S. (2013a), "Developing a computational model to understand the contributions of social learning modes to task coordination in teams", Artificial Intelligence for Engineering Design, Analysis and Manufacturing (AIEDAM), Vol. 27 No. 1, pp. 3-17. https://doi.org/10.1017/S0890060412000340.

Singh, V., Dong, A. and Gero, J.S. (2013b), "Computational studies to understand the role of social learning in team familiarity and its effects on team performance", CoDesign, Vol. 8, No. 1, pp. 25-41. https://doi.org/10.1080/15710882.2011.633088

Stacey, M., Eckert, C. and Earl, C. (2007), "From Ronchamp by sledge: On the pragmatics of object references", In: McDonnell, J. and Lloyd, P. (Eds.), About: designing - Analysing design meetings, Taylor and Francis, London, pp. 361-379.

Stempfle, J. and Badke-Schaub, P. (2002), “Thinking in design teams: An analysis of team communication”, Design Studies, Vol. 23 No. 5, pp. 473-496. https://doi.org/10.1016/S0142-694X(02)00004-2 
Wegner, D.M. (1995), "A computer network model of human transactive memory”, Social Cognition, Vol. 13, pp. 1-21. https://doi.org/10.1521/soco.1995.13.3.319

Dr. Vishal Singh, Assistant Professor

Aalto University, Civil Engineering

Rakentajanaukio 4A, 02150 Espoo, Finland

Email: vishal.singh@aalto.fi 